\title{
Growth of polymer-metal nanocomposites by pulsed laser deposition
}

\author{
Johanna Röder · Jörg Faupel · Hans-Ulrich Krebs
}

Received: 12 October 2007 / Accepted: 9 April 2008 / Published online: 20 June 2008

(C) The Author(s) 2008. This article is published with open access at Springerlink.com

\begin{abstract}
Complex polymer-metal nanocomposites have a wide range of applications, e.g. as flexible displays and packaging materials. Pulsed laser deposition was applied to form nanostructured materials consisting of metal clusters $(\mathrm{Ag}, \mathrm{Au}, \mathrm{Pd}$ and $\mathrm{Cu}$ ) embedded in a polymer (polycarbonate, PC) matrix. The size and amount of the metal clusters are controlled by the number of laser pulses hitting the respective targets. For $\mathrm{Cu}$ and Pd, smaller clusters and higher cluster densities are obtained as in the cases of $\mathrm{Ag}$ and $\mathrm{Au}$ due to a stronger reactivity with the polymers and thus a lower diffusivity. Implantation effects, differences in metal diffusivity and reactivity on the polymer surfaces, and the coalescence properties are discussed with respect to the observed microstructures on PC and compared to the metal growth on poly (methyl methacrylate), PMMA.
\end{abstract}

PACS 68.55.-a $\cdot 81.15 . \mathrm{Fg} \cdot 82.35 . \mathrm{Np}$

\section{Introduction}

Complex nanostructured materials consisting of polymers and metals have a wide range of applications, for instance in the form of electronic devices, flexible displays and packaging materials [1-3]. With the combination of such different materials one can merge the mechanical flexibility of polymers with special features, for instance optical absorption properties, which are correlated with the small dimensions of the metal clusters. In the case of thin-film deposition,

J. Röder (凶) · J. Faupel · H.-U. Krebs Institut für Materialphysik, University of Göttingen, Friedrich-Hund-Platz 1, 37077 Göttingen, Germany e-mail: jroeder@ump.gwdg.de metal clusters can be grown by the agglomeration of atoms deposited on the substrate surface, known as Vollmer-Weber island growth [4]. The cluster size and shape are defined by the growth mechanisms on the substrate surface, which strongly depend on the preparation conditions and on the diffusivity of the metal in the used matrix and on its surface. In this way metal clusters were for instance already grown on polyimide or Teflon by thermal evaporation [5] and on poly (methyl methacrylate), PMMA, by pulsed laser deposition (PLD) [6].

The aim of this paper is to study the morphology of metal clusters $(\mathrm{Ag}, \mathrm{Au}, \mathrm{Pd}$ and $\mathrm{Cu}$ ) embedded in polycarbonate (PC) as well as their optical properties. Additionally, the observed metal nanostructures are compared to those grown in a PMMA matrix. Finally, the growth mechanisms leading to the observed nanostructures are investigated in these regards, focussing especially on implantation effects, differences in metal diffusivity and reactivity with the polymer. Further, we will discuss whether the clusters are formed on the substrate surface or below due to implantation effects.

\section{Experimental setup}

Polymer-metal composites consisting of metal clusters (Ag, $\mathrm{Au}, \mathrm{Pd}$ and $\mathrm{Cu}$ ) embedded in a PC matrix were deposited on $\mathrm{Si}(111)$ substrates or $\mathrm{Cu}$ grids (3.05-mm diameter, 200 meshes) at room temperature by PLD. For this, a $\mathrm{KrF}$ excimer laser with a wavelength of $248 \mathrm{~nm}$, pulse duration of $30 \mathrm{~ns}$ and repetition rate of $10 \mathrm{~Hz}$ was used. PC and the metals were deposited at laser fluences of $70 \mathrm{~mJ} / \mathrm{cm}^{2}$ and 4-6 J/ $\mathrm{cm}^{2}$, respectively, in an ultra-high-vacuum chamber with a base pressure of about $10^{-8}$ mbar. The thicknesses of the polymer and metal layers are given by the number of laser pulses used. The average deposition rate of 
the metal was determined on Si substrates by profilometry. The target-to-substrate distance was $50 \mathrm{~mm}$. The setup has been described in more detail earlier [7]. The microstructure of the nanocomposites was investigated by transmission electron microscopy (TEM, Philips EM400). For these observations, the films were deposited directly onto $\mathrm{Cu}$ grids. Atomic force microscopy (AFM) measurements were performed in air using a Digital Instruments NanoScope 4 in tapping mode. UV-Vis spectra were taken by a Varian Cary 50 spectrometer. In this case, the samples were deposited on $\mathrm{Al}_{2} \mathrm{O}_{3}$ substrates. SRIM simulations were done using the program version 2003.26 [8]. The experimental details of the PMMA-metal samples were described earlier [6].

\section{Results and discussion}

For all studies, first an about 20-nm-thick polycarbonate film was deposited at a laser fluence of $70 \mathrm{~mJ} / \mathrm{cm}^{2}$. The deposition leads to smooth films with a surface roughness of about $0.1 \mathrm{~nm}$ and only a small number of droplets [9] as typically for the PLD process [10]. On these polymer surfaces different metals ( $\mathrm{Ag}, \mathrm{Au}, \mathrm{Pd}$ and $\mathrm{Cu}$ ) were deposited at a laser fluence of 4-6 J/cm ${ }^{2}$ with average film thicknesses of less than $5 \mathrm{~nm}$. The obtained metal film thickness was proportional to the number of laser pulses used for the deposition. In some cases, a final PC layer was deposited afterwards to embed the metal clusters deep in the polymer.

First, the growth of $\mathrm{Ag}$ on PC is described. In Fig. 1, as an overview two TEM images of Ag clusters deposited on
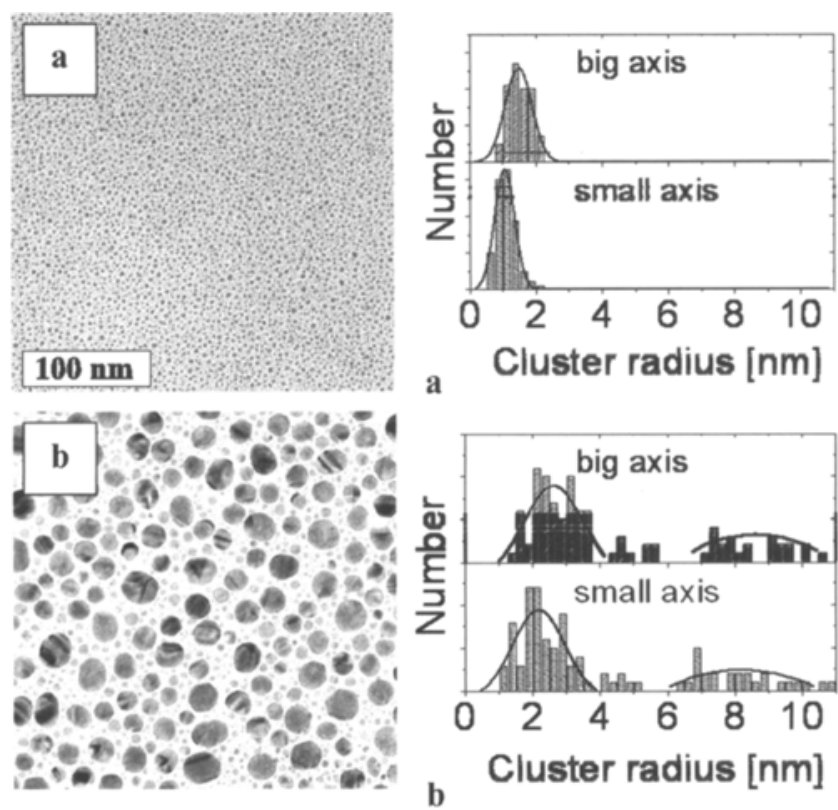

Fig. 1 TEM images of Ag between two 20-nm-thick PC layers with the respective size distributions. Average metal amounts: (a) $0.3 \mathrm{~nm}$, (b) $4.7 \mathrm{~nm}$
PC are shown. All films exhibit separated clusters. With increasing number of laser pulses the cluster size increases, while the cluster density decreases as soon as coalescence processes occur. Although the clusters show more or less spherical shapes, one can observe an increasing amount of elliptical clusters above a thickness of about $1 \mathrm{~nm}$. To determine the size distribution of the embedded clusters, the TEM pictures were analyzed using the software DEPP [11] by fitting ellipses to the clusters and calculating the small and big axes of the ellipses. The resulting size distributions for $\mathrm{Ag}$ films on PC with average Ag thicknesses of 0.5 and $4.7 \mathrm{~nm}$, respectively, are also given in Fig. 1. Additionally, one or rather two Gaussian curves were fitted to the graphs to determine the average cluster sizes and size distributions. For smaller amounts of metal an average cluster radius of about 1-2 $\mathrm{nm}$ was observed, whereas for thicker films the clusters show a bimodal size distribution as already observed for $\mathrm{Ag}$ on PMMA [6]. Many large grains exist with a radius of between 6 and $10 \mathrm{~nm}$, but additionally in the spaces in between a large number of small clusters with radii of about $2 \mathrm{~nm}$ are visible. This indicates that coalescence and secondary nucleation take place simultaneously in between the clusters. As can be seen in the different contrasts, the biggest particles consist of several grains with different orientations. The TEM diffraction patterns (not shown here) indicate that the clusters are randomly orientated and obey fcc structure.

Figure 2 summarizes the results on the cluster radius vs. layer thickness of $\mathrm{Ag}$ and vs. the number of laser pulses used. Up to a layer thickness of about $2 \mathrm{~nm}$, a linear increase of the cluster size occurs, while above this layer thickness the bimodal size distribution is observed. Since the dimensions of the big clusters continuously increase with the deposited film thickness, their size can be controlled by the number of laser pulses. In contrast, the small clusters in be-

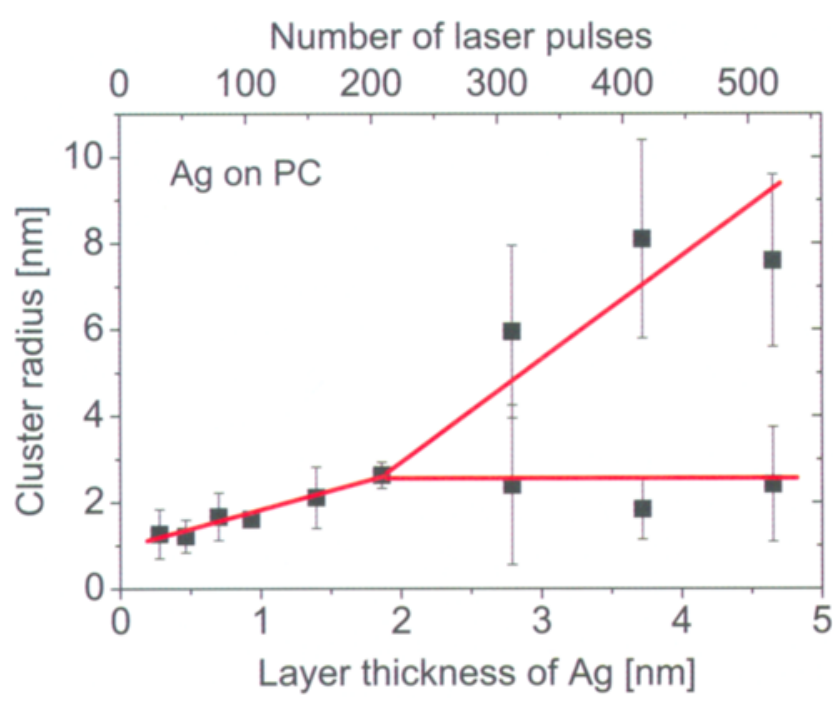

Fig. 2 Average cluster radii of the larger and smaller Ag grains vs. layer thickness 




Fig. 3 TEM images of different metals between two 20-nm-thick PC layers with average metal amounts of (a) $7.3 \mathrm{~nm}$ for $\mathrm{Au}$, (b) $3.3 \mathrm{~nm}$ for $\mathrm{Pd}$, (c) $3.8 \mathrm{~nm}$ for $\mathrm{Cu}$ and (d) $3.3 \mathrm{~nm}$ for $\mathrm{Pd}$

tween have always sizes in the range of only a few nanometers.

On a quick view the results of Au clusters in PC are similar to those of Ag in PC (see Fig. 3a). Also, Au grows in fcc structure with statistically oriented clusters. Again, the cluster size increases with increasing layer thickness (not shown here, see [9]) and a bimodal size distribution is observed for larger thicknesses. But, most particles are not spherical and show facetted surfaces. In contrast to $\mathrm{Ag}$ and $\mathrm{Au}$, the morphology of $\mathrm{Pd}$ and $\mathrm{Cu}$ exhibits more and smaller clusters (see Fig. 3b, c). In both cases, no bimodal growth is observed and the clusters are more irregularly formed. The high-resolution TEM image of Fig. 3d clearly shows that Pd clusters also have facetted surfaces.

To investigate the influence of the polymer, metal clusters were also deposited on PMMA and the morphology was compared with the growth on PC. In general the cluster shapes show a similar behaviour on both polymers as can be seen in Fig. 4 for $\mathrm{Ag}$ and $\mathrm{Cu}$ films. In the case of $\mathrm{Ag}$ on PMMA again a transition from non-bimodal to bimodal growth can be observed with increasing metal amount [6]. Compared to the growth on PC the cluster density is slightly reduced, the size distribution is narrower and the distances between the clusters are increased, thus indicating a slightly better diffusivity than on PC. In contrast to that, $\mathrm{Cu}$ has a similar cluster density, again no bimodal growth and mostly non-spherical, facetted particles.

In Fig. 5 it is shown for Ag on PC that the maximum of the optical absorption can be tuned by the cluster size and distribution. With increasing Ag content, the maximum of
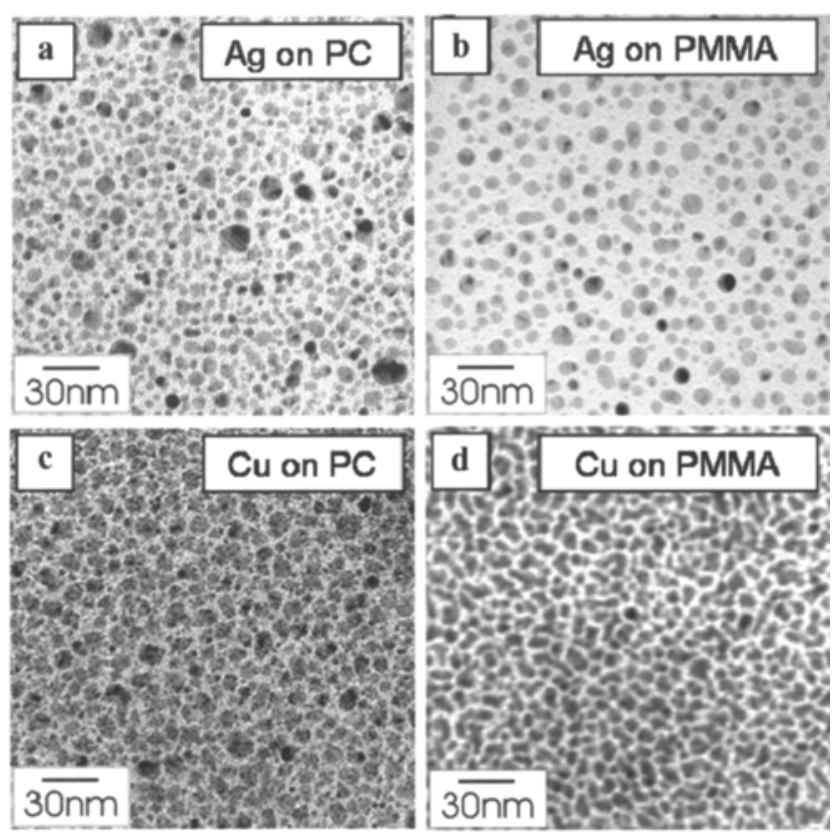

Fig. 4 TEM images of $\mathrm{Ag}$ and $\mathrm{Cu}$ clusters grown between two 20-nm-thick PC layers and on 15-nm PMMA, respectively, with metal amounts of (a) $1.9 \mathrm{~nm}$ for $\mathrm{Ag}$ on PC, (b) $2.0 \mathrm{~nm}$ for Ag on PMMA, (c) $3.8 \mathrm{~nm}$ for $\mathrm{Cu}$ on $\mathrm{PC}$ and (d) $3.6 \mathrm{~nm}$ for $\mathrm{Cu}$ on PMMA

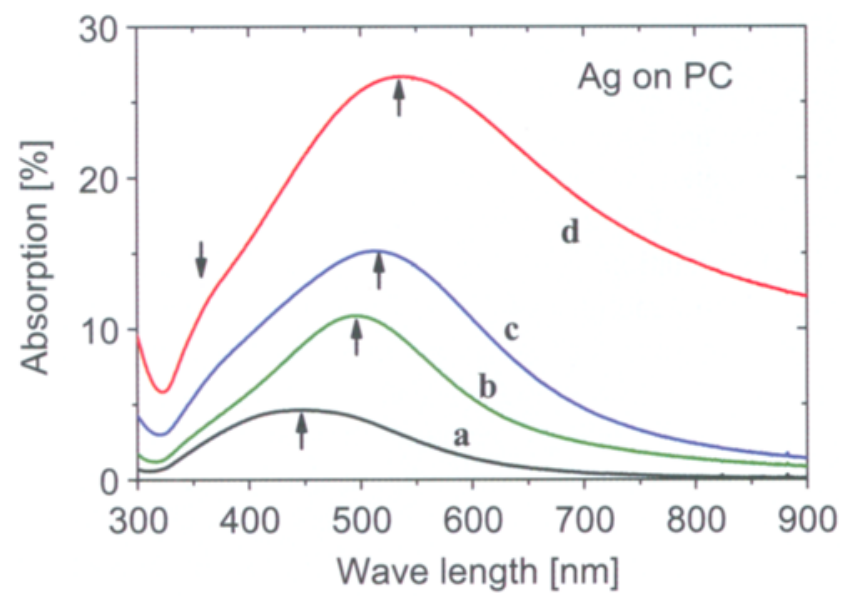

Fig. 5 Absorption spectra of Ag layers embedded in 40-nm-thick PC with Ag layer thicknesses of (a) $0.3 \mathrm{~nm}$, (b) $0.9 \mathrm{~nm}$, (c) $1.9 \mathrm{~nm}$ and (d) $4.7 \mathrm{~nm}$

the absorption is shifted from $450 \mathrm{~nm}$ at a cluster radius of about $1 \mathrm{~nm}$ to larger wavelengths. For the thickest sample with a bimodal size distribution the main absorption occurs at a wavelength of about $550 \mathrm{~nm}$, but also a second peak in the form of a shoulder in the spectrum is observed.

In the following, we try to understand the observed microstructures of the metal clusters and their morphologies in the different systems. With respect to the questions discussed it is important to remember that the pulsed laser deposition of metals in ultra-high vacuum at the used laser conditions is a combination of the deposition of metal atoms 


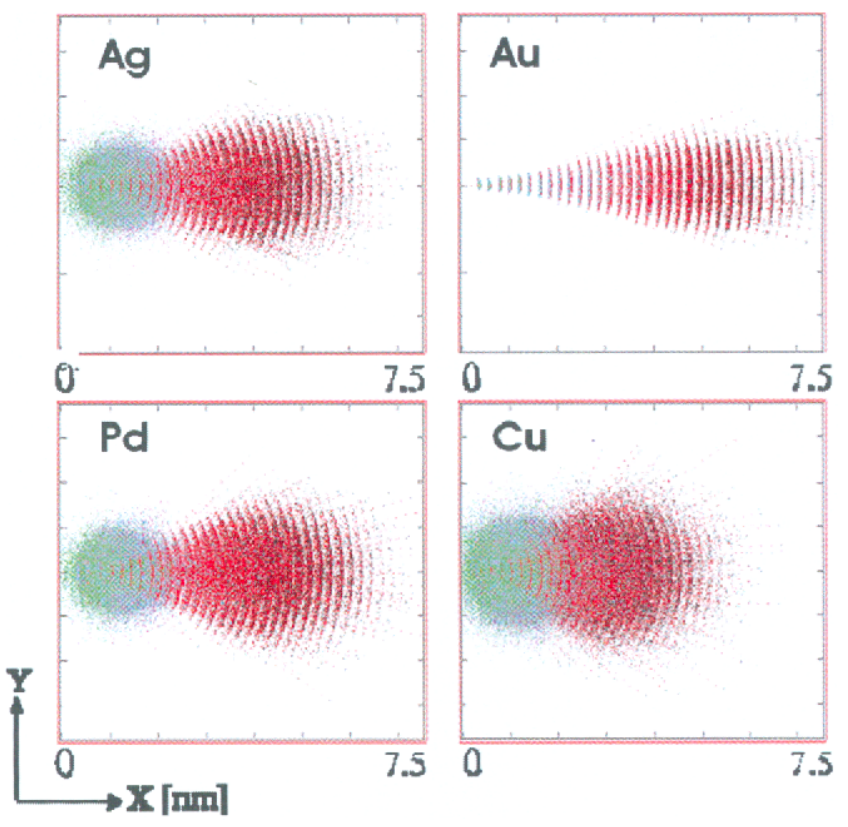

Fig. 6 SRIM simulations of the implantation of different energetic metal ions in PMMA. The simulations were done using 25000 metal ions hitting the PMMA surface at $x=0$ with kinetic energies of $100 \mathrm{eV}$. In the figure, the projection onto the $x y$ plane is shown. Coloured are the ion tracks (red) and the moved polymer atoms (other colours)

with energies of about $5-10 \mathrm{eV}$ and ions (50\% and more) with average energies of about $100 \mathrm{eV}$ [12]. While the atoms are deposited on the polymer surface, the energetic ions are implanted below the substrate surface. This can be seen in SRIM simulations of the implantation of 25000 metal ions with kinetic energies of $100 \mathrm{eV}$ into PMMA and PC. The results are given in Fig. 6 for the different metals used on PMMA. The ions impinging on the polymer surface at $x=0$ are implanted a few nm deep into the material. For PMMA, the calculated average implantation depths are $4.0 \mathrm{~nm}$ for $\mathrm{Ag}, 4.9 \mathrm{~nm}$ for $\mathrm{Au}, 4.3 \mathrm{~nm}$ for $\mathrm{Pd}$ and $3.1 \mathrm{~nm}$ for $\mathrm{Cu}$, respectively (with an error of about $0.6 \mathrm{~nm}$ ). In the case of $\mathrm{PC}$, slightly lower values of $3.8 \mathrm{~nm}$ for $\mathrm{Ag}, 4.7 \mathrm{~nm}$ for $\mathrm{Au}$, $4.1 \mathrm{~nm}$ for Pd and $3.0 \mathrm{~nm}$ for $\mathrm{Cu}$ are obtained. It is interesting to note that in contrast to the deposition of metals on metals, where sputtering yields of more than 0.5 were observed [13], the resputtering effects are negligible (less than $0.2 \%$ for all the metals used) in the case of the deposition of metals on both polymers.

Diffusion processes on and within the polymer material then lead to the formation of clusters by Vollmer-Weber island formation and growth. Because of the large difference in the surface energy it is expected that the metal islands are spherical. With increasing number of laser pulses during deposition, these islands grow until finally two islands hit each other and coalescence processes become important, leading to the different nanostructures. These can be explained by different diffusivities of the metals on and in the
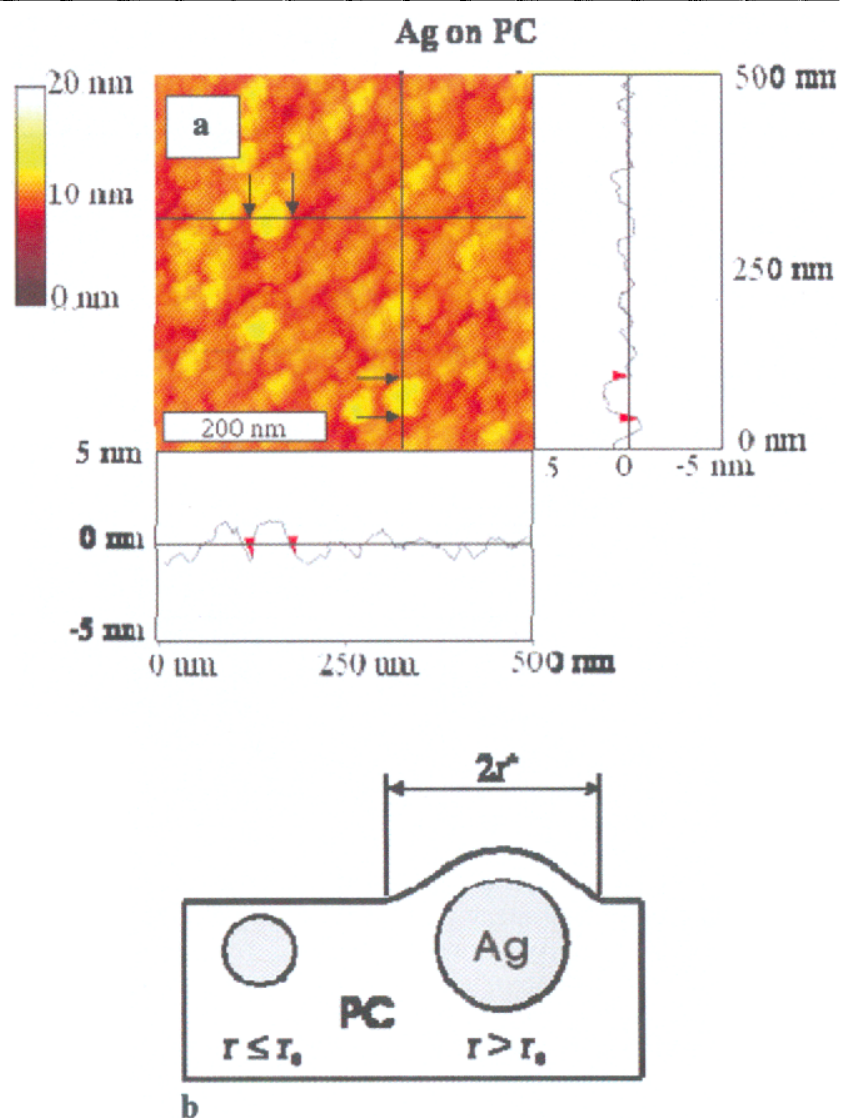

Fig. 7 AFM image after deposition of $\mathrm{Ag}$ (thickness of $4.7 \mathrm{~nm}$ ) on 20-nm PC, without top layer (a), and schematic drawing of Ag clusters below the PC surface in side view leading to a larger hill diameter $2 r^{*}$ compared to the size of the metal clusters (b)

polymer leading to changes in the nucleation, growth and coalescence processes (e.g. [14]). Fewer clusters with larger distances are formed by noble metals like $\mathrm{Ag}$ or $\mathrm{Au}$ than by a worse diffuser like $\mathrm{Pd}$ or $\mathrm{Cu}$. As soon as two growing clusters touch each other during growth by accumulation of atoms, coalescence starts via surface and interphase diffusion. Therefore, total coalescence of two grains hitting each other during growth needs time, the so-called coalescence time $\tau_{\text {coal }} . \tau_{\text {coal }}$ strongly depends on the diffusivity $D_{S}$, the radius $R$ of the clusters as well as the surface temperature $T[14]$, and is given by

$\tau_{\text {coal }}=\frac{k T \cdot R^{4}}{D_{S} \cdot \gamma \cdot \Omega^{4 / 3}}$,

with the Boltzmann factor $k$, the surface energy $\gamma$ and the atom size $\Omega$. In the cases of $\mathrm{Ag}$ and $\mathrm{Au}$, on both polymers total coalescence is obtained up to large cluster sizes due to a comparatively fast diffusion. Additionally, secondary nucleation occurs in the depletion zones in between the grains and, as a consequence, a bimodal distribution of clusters is formed as soon as a certain amount of metal (at a layer thickness of about $2 \mathrm{~nm}$ for $\mathrm{Ag}$ ) is exceeded. Monte Carlo simu- 
lations made by Carrey and Maurice [15] agree that such a microstructure with bimodal size distribution is obtained by very fast diffusion of the metal and non-instantaneous coalescence. The different morphologies of $\mathrm{Pd}$ on $\mathrm{PC}$ or $\mathrm{Cu}$ on PC and PMMA are due to the fact that these metal atoms react more easily with the polymer than $\mathrm{Ag}$ or $\mathrm{Au}$, and thus have a lower diffusivity and a lower mean free path on the polymer surface. Therefore, clusters with small distances are obtained. Total coalescence of touching clusters is impeded due to the slow diffusion and comparatively large coalescence times.

From the TEM investigations alone it remains unclear whether the metal clusters are formed on the polymer surface or are generated below the surface directly by the implanted ions. But, when comparing the TEM results of Fig. 1b (Ag on PC) with AFM measurements performed on a sample of the same run, but without top layer (see Fig. 7a), indications are given that the formed clusters lie below the polymer surface. The fundamental difference between these measurements is that the biggest clusters in the AFM measurements (marked with arrows) have small and big axes of about 15 and $25 \mathrm{~nm}$, respectively, while the TEM investigations show cluster radii of only up to $10 \mathrm{~nm}$. At the same time, the highest elevation in the AFM measurement is in the order of only $2.5 \mathrm{~nm}$, which is far below the cluster radius. Therefore, we conclude that the $\mathrm{Ag}$ clusters lie below the polymer surface as schematically depicted in Fig. 7b leading to a larger hill diameter $2 r^{*}$ compared to the real size $r$ of the metal clusters measured by TEM, as long as they are deeper below the PC surface than the distance $r_{0}$ of its centre. At this point it still remains unclear whether the clusters are directly formed below the polymer surface during deposition by the implanted ions or whether they grow first on the surface and then sink in due to the much lower surface energy of the polymer compared to the metal.

\section{Conclusion}

In summary, we have shown that complex nanostructured materials consisting of a polymer (PC or PMMA) and embedded metals (here $\mathrm{Ag}, \mathrm{Au}, \mathrm{Pd}$ and $\mathrm{Cu}$ ) can be grown by pulsed laser deposition in a well-controlled way. The deposition of metals on polymers is combined with implantation of energetic ions a few nanometers below the polymer surface. Diffusion processes and Vollmer-Weber island growth then lead to the formation of metal clusters which lie slightly below the polymer surface. The size of the metal clusters is controlled by the number of laser pulses. In the cases of $\mathrm{Ag}$ and $\mathrm{Au}$, a high diffusion of the metals is observed leading to more or less spherical particles with a bimodal size distribution caused by total coalescence and secondary nucleation processes. Compared to the results on PC, the Ag clusters show larger distances on PMMA, indicating a higher diffusivity of Ag on PMMA than on PC. For Pd and $\mathrm{Cu}$, a high reactivity exists with the polymer surface and the diffusion is decreased resulting in much smaller clusters and a higher number.

Acknowledgements This work was supported by the Deutsche Forschungsgemeinschaft (SFB 602 and GK 782).

Open Access This article is distributed under the terms of the Creative Commons Attribution Noncommercial License which permits any noncommercial use, distribution, and reproduction in any medium, provided the original author(s) and source are credited.

\section{References}

1. S.A. Van Slyke, C.H. Chen, C.W. Tan, Appl. Phys. Lett. 69, 2160 (1996)

2. A. Tsumura, H. Koezuka, T. Ando, Appl. Phys. Lett. 49, 1210 (1986)

3. K.L. Mittal (ed.), Metallized Plastics: Fundamentals and Applications (Dekker, New York, 1998)

4. M. Vollmer, A. Weber, Z. Phys. Chem. 119, 277 (1925)

5. A. Biswas, O.C. Aktas, U. Schürmann, U. Saeed, V. Zaporojtchenko, F. Faupel, T. Strunskus, Appl. Phys. Lett. 84, 2655 (2004)

6. J. Röder, H.U. Krebs, Appl. Phys. A 85, 15 (2006)

7. H.U. Krebs, J. Non-Equilib. Process. 10, 3 (1997)

8. The SRIM program. http://www.srim.org

9. J. Faupel, Ph.D. thesis, University of Göttingen, 2005

10. R. Eason (ed.), Pulsed Laser Deposition of Thin Films (Wiley, New York, 2007)

11. H. Voss, J. Ewerts, G. Schmitz, F. Hartung, Digital Esoteric Photo Processor, version 2.2.0 (1998)

12. S. Fähler, H.U. Krebs, Appl. Surf. Sci. 96-98, 61 (1996)

13. K. Sturm, H.U. Krebs, J. Appl. Phys. 90, 1061 (2001)

14. G. Jeffers, M.A. Dubson, P.M. Duxbury, J. Appl. Phys. 75, 5016 (1994)

15. J. Carrey, J.-L. Maurice, Phys. Rev. B 65, 205401 (2002) 